\title{
Remarks on a Mean Field Equation on $\mathrm{S}^{2}$
}

\author{
Changfeng Gui ${ }^{1}$, Fengbo Hang ${ }^{2, *}$, Amir Moradifam ${ }^{3}$ \\ and Xiaodong Wang ${ }^{4}$ \\ ${ }^{1}$ Department of Mathematics, University of Texas at San Antonio, San Antonio, \\ TX 78249, USA; \\ ${ }^{2}$ Courant Institute, New York University, New York, NY 10012, USA; \\ ${ }^{3}$ Department of Mathematics, University of California at Riverside, Riverside, \\ CA 92521, USA; \\ ${ }^{4}$ Department of Mathematics, Michigan State University, East Lansing, MI 48824, \\ USA.
}

Received May 27, 2019; Accepted September 23, 2019;

Published online January 12, 2021.

Dedicated to Professors Sun-Yung Alice Chang and Paul C. Yang on their 70th birthdays

\begin{abstract}
In this note, we study symmetry of solutions of the elliptic equation

$$
-\Delta_{\mathrm{S}^{2}} u+3=e^{2 u} \text { on } \mathrm{S}^{2},
$$

that arises in the consideration of rigidity problem of Hawking mass in general relativity. We provide various conditions under which this equation has only constant solutions, and consequently imply the rigidity of Hawking mass for stable constant mean curvature (CMC) sphere.
\end{abstract}

AMS subject classifications: 35J61, 58J05, 35B06

Key words: Semilinear elliptic equation, sphere covering inequality, rigidity of Hawking mass.

\section{Introduction}

The main aim of this note is to study the semilinear elliptic equation

$$
-\Delta_{\mathrm{S}^{2}} u+\alpha=e^{2 u}
$$

on the standard $S^{2}$. Here $u$ is a smooth function on $S^{2}$ and $\alpha$ is a positive constant.

*Corresponding author. Email addresses: changfeng.gui@utsa.edu (C. Gui), fengbo@cims.nyu.edu (F. Hang), moradifam@math.ucr.edu (A. Moradifam), xwang@math.msu.edu (X. Wang) 
When $\alpha=1$, (1.1) means that the conformal metric $e^{2 u} g_{S^{2}}$ has constant curvature 1 . Therefore all solutions are given by the pull back of the standard metric by Mobius transformations. This and more general statements also follow from the powerful method of moving plane (see [5,9]). The latter approach can be used to show (1.1) has only constant solution when $0<\alpha<1$ (see [16]). More recently, the sphere covering inequality was discovered in [12] and applied to show all solutions to equation (1.1) must be constant functions for $1<\alpha \leq 2$. In particular, this confirms a long-standing conjecture of ChangYang $([3,4])$ concerning the best constant in Moser-Trudinger type inequalities. Sphere covering inequality and its generalization can also be used to solve many uniqueness and symmetry problems from mathematical physics (see $[1,10,12]$ and many references therein). [10] explains the sphere covering inequality from the point view of comparison geometry and provides some further generalizations. In contrast, for $2<\alpha<3$, nontrivial axially symmetric solutions were found in [15]. The multiplicity of these nontrivial axially symmetric solutions was carefully discussed in [8]. More recently, non-axially symmetric solutions to (1.1) for $\alpha>4$ but close to 4 were found in [11]. In related developments, topological degree of (1.1) for $\alpha \notin \mathbb{Z}$ was computed in $[6,14,15]$. We refer the readers to the survey article [20] for more details of mean field equations on a closed surface.

Recently, $[18,19]$ discovered the interesting connection between the equation (1.1) with $\alpha=3$ and rigidity problems involving Hawking mass in general relativity. Among other results, it was shown in [18] that for $2<\alpha<4$, any even solution to (1.1) must be axially symmetric. In particular, when $\alpha=3$, any even solution, $u(x)=u(-x)$ for all $x \in \mathbb{S}^{2}$, must be a constant function. It is also conjectured in [18, Section 3] that for $2<\alpha \leq 3$, any solution to (1.1) must be axially symmetric. Our note is motivated by this conjecture. Our main result is

Theorem 1.1. Assume $2<\alpha \leq 3$ and $u \in C^{\infty}\left(\mathbb{S}^{2}\right)$ is a solution to

$$
-\Delta_{\mathrm{S}^{2}} u+\alpha=e^{2 u}
$$

If for some $p \in \mathbb{S}^{2}, \nabla u(p)=0$ and $D^{2} u(p)$ has two equal eigenvalues, then $u$ is axially symmetric with respect to $p$. In particular, in the case $\alpha=3, u$ must be a constant function.

We may call the point $p$ in the assumption as an umbilical critical point of $u$. So the theorem reads as: for $2<\alpha \leq 3$, any solution with an umbilical critical point must be axially symmetric with respect to that point. Here we do not know whether the solution is even or not. On the other hand, the approach to Theorem 1.1 can help us relax the even assumption in [18] a little bit. One typical example is

Theorem 1.2. Assume $2<\alpha \leq 3$ and $u \in C^{\infty}\left(\mathrm{S}^{2}\right)$ is a solution to

$$
-\Delta_{\mathrm{S}^{2}} u+\alpha=e^{2 u} \text {. }
$$

If every large circle splits $\mathbb{S}^{2}$ as two half sphere with equal area under the metric $e^{2 u} g_{S^{2}}$, then $u$ is axially symmetric with respect to some point. In particular, in the case $\alpha=3, u$ must be a constant function. 
Note that if $u$ is even, then any large circle clearly splits the area. In Section 3, we will also present several other conditions which is weaker than the even assumption (see Proposition 3.1, 3.2). It is unfortunate we are not able to remove any of these assumptions.

At last we point out that there is an analogous nonlocal problem on $S^{1}$, namely

$$
\begin{cases}\Delta u=0, & \text { on } B_{1}^{2} \subset \mathbb{R}^{2}, \\ \frac{\partial u}{\partial v}+\lambda=e^{u}, & \text { on } \mathbb{S}^{1} .\end{cases}
$$

Here $v$ is the unit outer normal direction and $\lambda$ is a positive constant. This equation appears in the study of determinant of Laplacian on compact surface with boundary (see [17]). The solutions to the above problem is well understood (see [17, Lemma 2.3] and [21, Theorem 3]). The reason the problem on $S^{1}$ is much simpler than (1.1) is because the Fourier analysis on $\mathbb{S}^{1}$ is much easier.

In Section 2, we will describe our main new observation and use it to derive Theorem 1.1. In Section 3, we will apply this new observation to derive several relaxation of the even assumption in [18]. In particular, Theorem 1.2 will be proved.

\section{Proof of Theorem 1.1}

Let us fix the notations.

$$
\mathrm{S}^{2}=\left\{x \in \mathbb{R}^{3}: x_{1}^{2}+x_{2}^{2}+x_{3}^{2}=1\right\} .
$$

$e_{1}=(1,0,0), e_{2}=(0,1,0)$ and $e_{3}=(0,0,1)$ denotes the standard base. For $x, y \in \mathbb{R}^{3}, x \cdot y$ denotes the usual dot product. For any $y \in \mathbb{S}^{2}$, we write

$$
H_{y}=\left\{x \in \mathbb{S}^{2}: x \cdot y \geq 0\right\}
$$

as the half sphere, and

$$
C_{y}=\left\{x \in \mathbb{S}^{2}: x \cdot y=0\right\}
$$

as the large circle. We also denote $R_{y}$ as the reflection with respect to plane $\left\{x \in \mathbb{R}^{3}: x \cdot y=0\right\}$ i.e.,

$$
R_{y} x=x-2(x \cdot y) y .
$$

Assume $u \in C^{\infty}\left(S^{2}, \mathbb{R}\right)$ satisfies (1.1) with $2<\alpha \leq 3$, then

$$
\int_{\mathrm{S}^{2}} e^{2 u} d \mu=4 \pi \alpha .
$$

Here $\mu$ is the measure associated with standard metric.

Fix $y \in \mathbb{S}^{2}$, we define $v(x)=u\left(R_{y} x\right)$ for any $x \in \mathbb{S}^{2}$ and $w=u-v$. Then

$$
\left\{\begin{array}{l}
-\Delta_{S^{2}} w=e^{2 u}-e^{2 v}=c w \\
\left.w\right|_{C_{y}}=0 .
\end{array}\right.
$$


Here

$$
c(x)=2 \int_{0}^{1} e^{2((1-t) v(x)+t u(x))} d t
$$

is a smooth function on $S^{2}$.

If $w$ is not identically zero, then classical results (see $[2,7,13]$ ) imply that the nodal set of $w$ consists of finitely many smooth curves which only intersects at critical points of $w$. Moreover $w$ behaves like a harmonic polynomial near each critical point, i.e. nodal set locally looks like straight lines with equal angles at critical points.

If $\Omega \subset H_{y}$ is a simply connected nodal domain, then it follows from the sphere covering inequality ([12, Theorem 1.1]), or more precisely, the formulation with standard $S^{2}$ as background metric ( [10, Proposition 3.1]), that

$$
\int_{\Omega \cup R_{y}(\Omega)} e^{2 u} d \mu=\int_{\Omega} e^{2 u} d \mu+\int_{\Omega} e^{2 v} d \mu>4 \pi
$$

This inequality and (2.5) implies $H_{y}$ can not contain 3 or more simply connected nodal domains.

The crucial step to prove symmetry of solutions in $[12,18]$ is counting the number of simply connected nodal domains. As observed in [12, Section 4.2], if we have a critical point of $u$ on $C_{y}$, namely $q \in C_{y}$, and $w$ is not identically zero, then the order of $w$ at $q$ (i.e. the order of the first nonvanishing term in Taylor expansion of $w$ at $q$ ) is at least 2. Hence in $H_{y}$, at least one nodal line emanates from $q$ with equal angle. This implies $H_{y}$ contains at least two simply connected nodal domains.

Let $z$ be the unit tangent vector of $C_{y}$ at $q$. Our new observation is: if $z$ is an eigenvector of $D^{2} u(q)$, and $w$ is not identically zero, then the order of $w$ at $q$ is at least 3 . If the order is larger than or equal to 4 , then $H_{y}$ contains at least 3 simply connected nodal domains, and it contradicts with (2.5). When the order of $w$ at $q$ is 3 , the nodal set of $w$ emanates two lines from $q$ with angle $\frac{\pi}{3}$ in between, and $w$ takes alternating signs in each angle. Since we can not have 3 or more simply connected nodal domains, the only possibility is we have only two nodal domains (i.e. the two emanating nodal line from $q$ form a loop in $H_{y}$ ). It follows from Hopf principle that $\frac{\partial w}{\partial v}$ is nonzero and of a fixed sign on $C_{y} \backslash\{q\}$, here $v$ is the unit outer normal vector of $H_{y}$ (in fact, $v=-y$ ). In particular, there is no critical point on $C_{y} \backslash\{q\}$. We state this conclusion as a lemma.

Lemma 2.1. Assume $u$ is a smooth solution to (1.1) with $2<\alpha \leq 3$. Let $q$ be a critical point of $u$ and $z \in T_{q} S^{2}$ be an unit eigenvector of $D^{2} u(q)$. Denote $y=q \times z$ (the cross product in $\left.\mathbb{R}^{3}\right)$, $v=u \circ R_{y}$ and $w=u-v$. If $w$ is not identically zero, then $\frac{\partial w}{\partial v}$ is nonzero and of a fixed sign on $C_{y} \backslash\{q\}$, here $v=-y$ is the unit outer normal vector of $H_{y}$. Note that on $C_{y}, \frac{\partial w}{\partial v}=2 \frac{\partial u}{\partial v}$. Hence $\frac{\partial u}{\partial v}$ is nonzero and of a fixed sign on $C_{y} \backslash\{q\}$.

With Lemma 2.1 at hand, we are ready to derive Theorem 1.1. 
Proof of Theorem 1.1. By rotation we can assume the umbilical critical point $p=e_{3}$. It follows from [18, Lemma 5] that

$$
\int_{S^{2}} x_{i} u(x) d \mu(x)=0
$$

for $i=1,2,3$. Since every nonzero vector in $T_{e_{3}} S^{2}$ is an eigenvector of $D^{2} u\left(e_{3}\right)$, we know for every $y \in C_{e_{3}}$, we can apply Lemma 2.1 with $q=e_{3}$.

If $\nabla u\left(-e_{3}\right)=0$, then for any $y \in C_{e_{3}}, u \circ R_{y}=u$ (i.e. $u$ is symmetric with respect to $C_{y}$ ). Hence $u$ is axially symmetric with respect to $e_{3}$.

Next we claim $\nabla u\left(-e_{3}\right)$ must be zero. In fact, if this is not the case. Since $u$ must have another critical point besides $e_{3}$, by rotation we can assume it lies in $C_{e_{2}} \backslash\left\{e_{3}\right\}$. It follows from Lemma 2.1 that $u$ is symmetric with respect to $C_{e_{2}}$. For $0<\theta<\pi$, we know $u$ can not be symmetric with respect to $C_{-\sin \theta \cdot e_{1}+\cos \theta \cdot e_{2}}$ (because otherwise we conclude

$$
\nabla u\left(-e_{3}\right) \cdot e_{2}=0
$$

and

$$
\nabla u\left(-e_{3}\right) \cdot\left(-\sin \theta \cdot e_{1}+\cos \theta \cdot e_{2}\right)=0,
$$

it follows that $\nabla u\left(-e_{3}\right)=0$, a contradiction). Hence we know $\frac{\partial u}{\partial v}$ does not vanish on $C_{-\sin \theta \cdot e_{1}+\cos \theta \cdot e_{2}} \backslash\left\{e_{3}\right\}$ and it has a fixed sign. Here $v=\sin \theta \cdot e_{1}-\cos \theta \cdot e_{2}$. By continuity we know the sign also does not depend on $\theta$. It follows that for $-1 \leq s<1, u\left(\sqrt{1-s^{2}} \cos \theta\right.$, $\left.\sqrt{1-s^{2}} \sin \theta, s\right)$ is strictly monotone in $\theta \in(0, \pi)$. This contradicts with the fact

$$
\int_{S^{2}} x_{1} u(x) d \mu(x)=0
$$

Hence $\nabla u\left(-e_{3}\right)$ must be zero.

If $\alpha=3$, it follows from the fact $u$ is axially symmetric and [18, Proposition 1] that $u$ must be a constant function.

\section{Some relaxation of even assumption}

Here we want to show the discussion in Section 2 can help us relax the even assumption in [18].

Proof of Theorem 1.2. Note that the equal area assumption can be written as: for any $y \in \mathrm{S}^{2}$,

$$
\int_{H_{y}} e^{2 u} d \mu=\int_{H_{-y}} e^{2 u} d \mu
$$

Assume $q$ is a critical point of $u$ and $z \in T_{q} S^{2}$ is an eigenvector of $D^{2} u(q)$. Denote $y=q \times z$, $v=u \circ R_{y}$ and $w=u-v$. Then $w$ must be identically zero i.e. $u$ is symmetric with respect 
to $C_{y}$. Indeed if $w$ is not identically zero, it follows from Lemma 2.1 that $\frac{\partial w}{\partial v}$ is nonzero and of a fixed sign on $C_{y} \backslash\{q\}$, here $v=-y$ is the unit outer normal vector of $H_{y}$. Using

$$
-\Delta_{\mathrm{S}^{2}} w=e^{2 u}-e^{2 v},
$$

we see

$$
\int_{H_{y}} e^{2 u} d \mu-\int_{H_{-y}} e^{2 u} d \mu=\int_{H_{y}} e^{2 u} d \mu-\int_{H_{y}} e^{2 v} d \mu=-\int_{H_{y}} \Delta_{S^{2}} w d \mu=-\int_{C_{y}} \frac{\partial w}{\partial v} d s \neq 0 .
$$

This contradicts with the equal area assumption.

By rotation we can assume $e_{3}$ is a critical point of $u$, and $D^{2} u\left(e_{3}\right)$ has $e_{1}, e_{2}$ as eigenvectors. It follows from previous discussion that $u$ is symmetric with respect to $C_{e_{1}}$ and $\mathrm{C}_{e_{2}}$.

We will show $u$ must be symmetric with respect to $C_{e_{3}}$. One this is known it follows from [18, Lemma 8] that $u$ must be axially symmetric.

To continue we let $v=u \circ R_{e_{3}}$ and $w=u-v$, then using the equal area assumption, same argument as above shows

$$
\int_{C_{e_{3}}} \frac{\partial w}{\partial e_{3}} d s=0=2 \int_{C_{e_{3}}} \frac{\partial u}{\partial e_{3}} d s
$$

Hence $\frac{\partial u}{\partial e_{3}}$ vanishes at some point on $C_{e_{3}}$.

If $\frac{\partial u}{\partial e_{3}}\left(e_{1}\right)=0$, then it follows from the fact $u$ is symmetric with respect to $C_{e_{2}}$ that $\nabla u\left(e_{1}\right)=0$ and $D^{2} u\left(e_{1}\right)$ has $e_{2}, e_{3}$ as eigenvectors. On the other hand, it follows from the fact $u$ is symmetric with respect to $C_{e_{1}}$ that $\nabla u\left(-e_{1}\right)=0$. This together with Lemma 2.1 implies $u-u \circ R_{e_{3}}=0$ i.e. $u$ is symmetric with respect to $C_{e_{3}}$.

If $\frac{\partial u}{\partial e_{3}}\left(e_{2}\right)=0$, we can get symmetry with respect to $C_{e_{3}}$ exactly as above.

If $\frac{\partial u}{\partial e_{3}}(q)=0$ for some $q \in C_{e_{3}} \backslash\left\{ \pm e_{1}, \pm e_{2}\right\}$, we must have $w=u-u \circ R_{e_{3}}$ is identically zero. If it is not the case, then the order of $w$ at $q$ is at least 2 . By symmetry, the order of $w$ at $R_{e_{1}} q, R_{e_{2}} q$ and $-q$ must be at least 2 too. Hence $w$ has at least 3 simply connected nodal domain in $H_{e_{3}}$. This contradicts with the sphere covering inequality by the discussion in Section 2.

In all the cases, we know $u$ must be axially symmetric, and hence it must be constant when $\alpha=3$ ([18, Proposition 1]).

Along the same line we have the following

Proposition 3.1. Assume $2<\alpha \leq 3$ and $u \in C^{\infty}\left(\mathbb{S}^{2}\right)$ is a solution to

$$
-\Delta_{\mathrm{S}^{2}} u+\alpha=e^{2 u} \text {. }
$$

If there exists $p \in \mathbb{S}^{2}$ such that both $p$ and $-p$ are critical points of $u$, and

$$
\int_{H_{p}} e^{2 u} d \mu=\int_{H_{-p}} e^{2 u} d \mu
$$

then $u$ must be axially symmetric. If $\alpha=3$, then $u$ is a constant function. 
Proof. By rotation we can assume $p=e_{3}$ and $D^{2} u\left(e_{3}\right)$ has $e_{1}, e_{2}$ as eigenvector. It follows from Lemma 2.1 and the fact $\nabla u\left(-e_{3}\right)=0$ that $u$ is symmetric with respect to $C_{e_{1}}$ and $C_{e_{2}}$. Now using

$$
\int_{H_{e_{3}}} e^{2 u} d \mu=\int_{H_{-e_{3}}} e^{2 u} d \mu,
$$

the argument in the proof of Theorem 1.2 tells us $u$ is also symmetric with respect to $C_{e_{3}}$. It follows from [18, Lemma 8 ] that $u$ must be axially symmetric.

Proposition 3.2. Assume $2<\alpha \leq 3$ and $u \in C^{\infty}\left(\mathrm{S}^{2}\right)$ is a solution to

$$
-\Delta_{\mathrm{S}^{2}} u+\alpha=e^{2 u} \text {. }
$$

If there exists $p \in \mathbb{S}^{2}$ such that $\nabla u(p)=0, \nabla u(-p)=0$ and $D^{2} u(p)=D^{2} u(-p)$ (here we identify $T_{p} S^{2}$ with $T_{-p} S^{2}$ naturally), then $u$ must be axially symmetric. If $\alpha=3$, then $u$ is $a$ constant function.

Proof. By rotation we can assume $p=e_{3}$ and $D^{2} u\left(e_{3}\right)$ has $e_{1}, e_{2}$ as eigenvector. It follows from Lemma 2.1 and the fact $\nabla u\left(-e_{3}\right)=0$ that $u$ is symmetric with respect to $C_{e_{1}}$ and $C_{e_{2}}$. It follows from the equation that

$$
e^{2 u\left(e_{3}\right)}=-\Delta_{\mathrm{S}^{2}} u\left(e_{3}\right)+\alpha=-\Delta_{\mathrm{S}^{2}} u\left(-e_{3}\right)+\alpha=e^{2 u\left(-e_{3}\right)} .
$$

Hence $u\left(e_{3}\right)=u\left(-e_{3}\right)$. Let $w=u-u \circ R_{e_{3}}$, then because $w$ is symmetric with respect to $C_{e_{1}}$ and $C_{e_{2}}$, we see $w$ vanishes at least to order 4 (does not include 4 ) at $e_{3}$. If $w$ is not identically zero, then it will have at least 3 simply connected nodal domains. This contradicts with the sphere covering inequality by the discussion in Section 2. It follows from [18, Lemma 8] that $u$ must be axially symmetric.

\section{Acknowledgments}

C. Gui is partially supported by National Science Foundation Grant DMS-1601885. A. Moradifam is supported by National Science Foundation grant DMS-1715850. X. Wang is partially supported by Simons Foundation Collaboration Grant for Mathematicians no. 312820 .

\section{References}

[1] D. Bartolucci, C. Gui and A. Jevnikar et al., A singular sphere covering inequality: uniqueness and symmetry of solutions to singular Liouville-type equations, Math. Ann., 374(3/4) (2019), 1883-1922.

[2] L. Bers, Local behavior of solutions of general linear elliptic equations, Commun. Pure Appl. Math., 1955, 8 (1955), 473-496.

[3] S. Y. A. Chang and P. C. Yang, Prescribing Gaussian curvature on $\mathbb{S}^{2}$, Acta Math., 159(3/4) (1987), 215-259. 
[4] S. Y. A. Chang and P. C. Yang, Conformal deformation of metrics on $\mathrm{S}^{2}$, J. Differential Geom., 27(2) (1988), 259-296.

[5] W. Chen and C. Li, Classification of solutions of some nonlinear elliptic equations, Duke Math. J., 63(3) (1991), 615-622.

[6] C. Chen and C. Lin, Topological degree for a mean field equation on Riemann surfaces (English summary), Commun. Pure Appl. Math., 56(12) (2003), 1667-1727.

[7] S. Cheng, Eigenfunctions and nodal sets, Comment Math. Helv., 51(1) (1976), 43-55.

[8] J. Dolbeault, M. Esteban and G. Tarantello, Multiplicity results for the assigned Gauss curvature problem in $\mathbb{R}^{2}$ (English summary), Nonlinear Anal., 70(8) (2009), 2870-2881.

[9] B. Gidas, W. M. Ni and L. Nirenberg, Symmetry and related properties via the maximum principle, Commun. Math. Phys., 68(3) (1979), 209-243.

[10] C. Gui, F. Hang and A. Moradifam, The sphere covering inequality and its dual, arXiv: 1812.09994.

[11] C. Gui and Y. Hu, Non-axially symmetric solutions of a mean field equation on $\mathrm{S}^{2}$, arXiv:1709.02474.

[12] C. Gui and A. Moradifam, The sphere covering inequality and its applications, Invent. Math., 214(3) (2018), 1169-1204.

[13] P. Hartman and A. Wintner, On the local behavior of solutions of non-parabolic partial differential equations, Amer. J. Math., 75 (1953), 449-476.

[14] Y. Li, Harnack type inequality: the method of moving planes, Commun. Math. Phys., 200(2) (1999), 421-444.

[15] C. Lin, Topological degree for mean field equations on $S^{2}$, Duke Math. J., 104(3) (2000), 501536.

[16] C. Lin, Uniqueness of solutions to the mean field equations for the spherical Onsager vortex, Arch. Ration. Mech. Anal., 153(2) (2000), 153-176.

[17] B. Osgood, R. Phillips and P. Sarnak, Extremals of determinants of Laplacians, J. Funct. Anal., 80(1) (1988), 148-211.

[18] Y. Shi, J. Sun and G. Tian et al., Uniqueness of the mean field equation and rigidity of Hawking mass, Calc. Var. Partial Differential Equations, 58(2) (2019), Art. 41, 16 pp.

[19] J. Sun, Rigidity of Hawking mass for surfaces in three manifolds, Pacific J. Math., 292 (2018), 479-504.

[20] G. Tarantello, Analytical, geometrical and topological aspects of a class of mean field equations on surfaces (English summary), Discrete Contin. Dyn. Syst., 28(3) (2010), 931-973.

[21] X. Wang, Uniqueness results on surfaces with boundary, Calc. Var. Partial Differential Equations, 56(3) (2017), 87-97. 